\title{
OKP-Pflichtleistungen und Kostengutsprachen - (k)ein Widerspruch
}

\author{
Kostengutsprachen bieten Spitälern und Patienten Vergütungssicherheit. Für Ärzte \\ hingegen bedeutet es ein Mehr an administrativem Aufwand. Verzögerte oder nur \\ für kurze Zeit erteilte Kostengutsprachen sind für Ärzte und Patienten oft schwer \\ nachvollziehbar. An einer Veranstaltung der FMH-Fachkommission SwissDRG und \\ der FMH-Begleitgruppe ST Reha wurde nach Möglichkeiten zur Vereinfachung des \\ Kostengutsprache-Prozesses gesucht.
}

\section{Petra Ingenpass ${ }^{a}$ Gabriela Lang ${ }^{b}$}

a Dr. med., Stv. Leiterin Abteilung Tarife und Gesundheitsökonomie Spitalärzte

b Rechtsanwältin, Stv. Leiterin Abteilung Rechtsdienst FMH
Korrespondenz: FMH

Frohburgstrasse 15

CH-4600 Olten

Tel. 0313591111

Fax 0313591112

tarife.spital[at]fmh.ch

\section{Braucht es Kostengutsprachen bei OKP-Pflichtleistungen?}

Das Krankenversicherungsgesetz (KVG) geht grundsätzlich von der Vermutung aus, dass ärztliche Leistungen wirksam, zweckmässig sowie wirtschaftlich (WZW) sind und somit vom Krankenversicherer übernommen werden. Doch gibt es vertragliche und rechtliche Bestimmungen, die vor einem geplanten stationären Aufenthalt oder einer Rehabilitation eine Kostengutsprache (KoGu) vom Versicherer verlangen. Während diese für die Spitäler der finanziellen Absicherung dient, stellt sie für die Versicherer eine Kontrolle der WZW-Kriterien, insbesondere der Spital- bzw. Rehabedürftigkeit, dar [1]. Die Kostengutsprache bei ausserkantonaler Behandlung zeigt dem Patienten allfällige, nicht gedeckte Kosten auf. Auch für gewisse ambulante Leistungen sind KoGu notwendig, zum Beispiel nach vierzig Psychotherapien oder bei Behandlungen mit Onkologika im Off-Label-Use [2]. Für eine Rehabilitation werden die Kosten gemäss Krankenpflege-Leistungsverordnung (KLV) sogar nur nach «vorgängiger besonderer Gutsprache des Versicherers und mit ausdrücklicher Bewilligung des Vertrauensarztes» übernommen [3].

Für Ärzte ist der teils beträchtliche administrative Aufwand bei der Gesuchstellung sowie eine verzögerte Erteilung der Kostengutsprache durch den Versicherer problematisch. Auch in Fällen klarer Indikation zur stationären Rehabilitation wird eine KoGu oft nur für eine kurze Zeitspanne erteilt, so dass während einer laufenden Behandlung Verlängerungsgesuche notwendig sind - dies birgt Unsicherheiten für Patient und Arzt. Zudem werden Bearbeitungszeiten der Versicherer mit bis zu fünf Tagen oder mehr von den Ärzten und Patienten als unverhältnismässig lang erachtet.

\section{Rechtliche Grundlagen der KoGu für ärztliche Leistungen}

Während der Gesetzestext zur Invalidenversicherung festhält, dass vor jeder medizinischen Behand- lung eine Kostengutsprache nötigt ist [4], wird bei der Unfallversicherung die Klärung der KoGu den Tarifpartnern überlassen - Regelungen auf Gesetzesoder Verordnungsebene fehlen.

Im Krankenversicherungsgesetz ist eine Übernahme von WZW-konformen Leistungen durch den Versicherer geregelt. Der Bundesrat kann aber ärztliche Leistungen benennen, für die eine Kostenübernahme nicht oder nur unter bestimmten Bedingungen erfolgt [5]. Um die Qualität und den zweckmässigen Einsatz medizinischer Leistungen zu sichern, kann er vorsehen, dass bestimmte kostspielige diagnostische oder therapeutische Verfahren die $\mathrm{Zu}$ stimmung des Vertrauensarztes benötigen oder nur von dafür qualifizierten Leistungserbringern durchgeführt werden dürfen [6]. Der Bundesrat bzw. das EDI hat deshalb in der KLV (inkl. Anhang 1) ärztliche Leistungen aufgeführt, die von der OKP übernommen, nicht übernommen oder unter bestimmten Bedingungen übernommen werden. Die Aufzählung ist nicht abschliessend. Für bestimmte Leistungen wie z.B. Rehabilitationen wurden dabei hohe Hürden geschaffen.

\section{Raschere KoGu für Patienten wichtig}

Bei der von der gfs.bern im Auftrag der FMH durchgeführten Befragung gaben Ärzte an, dass die Versicherer KoGu oft verzögert erteilen [7]. Fristen zur Erteilung einer KoGu sind jedoch weder im Gesetz noch in den Verordnungen geregelt [8]. In den Tarifverträgen wurden unterschiedliche Regelungen vereinbart: von «so rasch wie möglich» bis «innerhalb fünf Tagen». Gemäss prozessualem Grundrecht muss jeder Entscheid eines Gerichts oder einer Verwaltungsbehörde binnen einer Frist, die «nach der Natur der Sache und nach den gesamten übrigen Umständen als angemessen erscheint», erfolgen [9]. An dieses Grundrecht sind auch die Versicherer gebunden, da sie mit der Durchführung der sozialen Krankenversicherung eine öffentliche Aufgabe des Bundes wahrnehmen [10]. Was aber heisst «ange- 


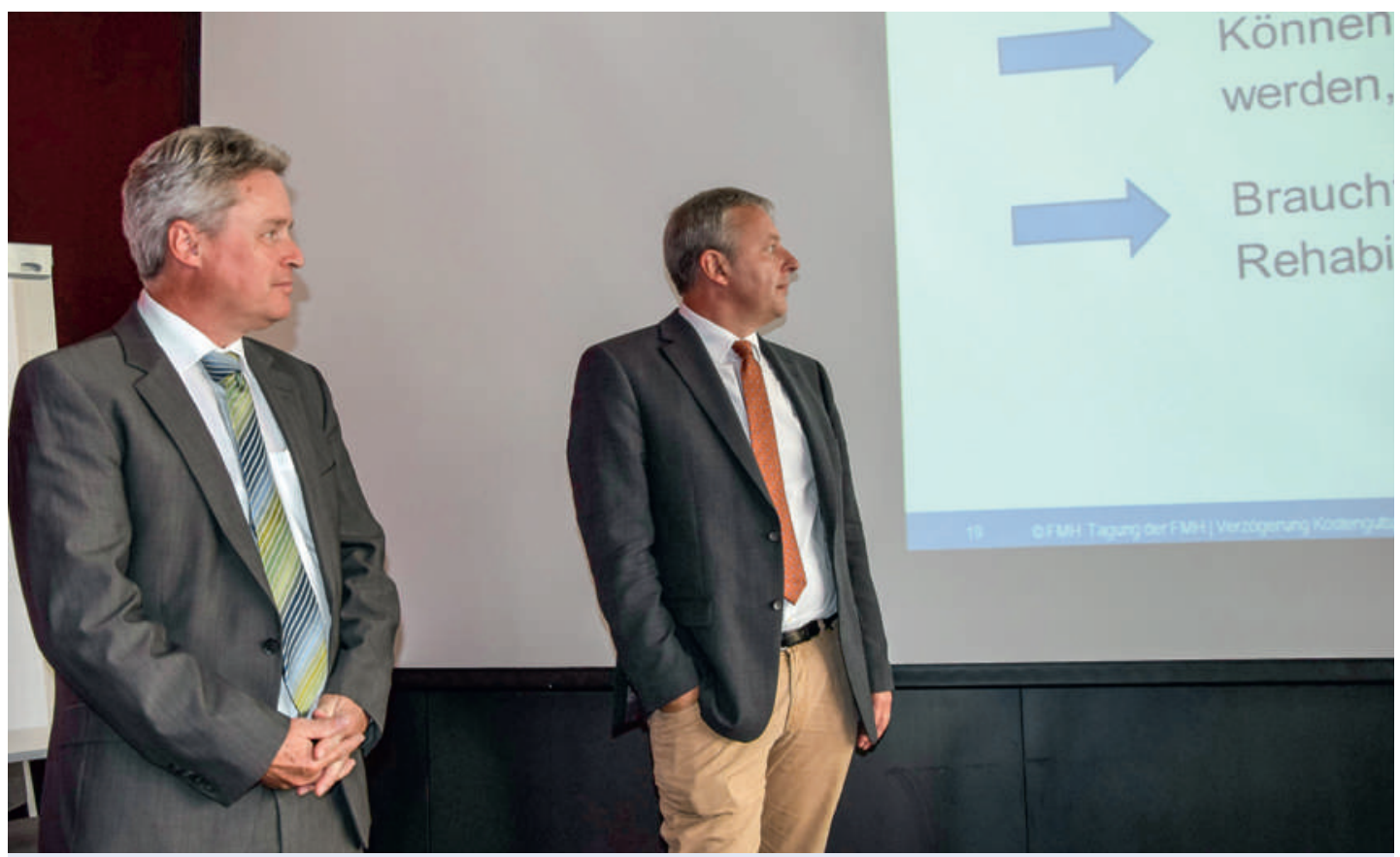

Stefan Wildi (rechts) und Beat Schärer stellen sich den Fragen zu «stillschweigenden Kostengutsprachen».

messen»? Nach bundesgerichtlicher Rechtsprechung wird der Grundsatz missachtet, wenn die Sache über Gebühr verschleppt wird. Die «angemessene» Dauer ist in jedem Einzelfall zu prüfen: Entscheidend sind die konkreten Umstände, Umfang und Komplexität der Sache [11]. Sogar wenn in den Tarifverträgen eine konkrete Frist für die Erteilung einer Kostengutsprache vereinbart ist, kann je nach gegebenem Fall eine kürzere, aber auch eine längere Frist als angemessen betrachtet werden.

\section{Kriterien zur Erteilung von KoGu}

Von den Fachgesellschaften wurden - oft gemeinsam mit den Vertrauens- und Versicherungsärzten bereits zahlreiche Empfehlungen erarbeitet: beispielsweise, wann nach Hüftgelenkersatz eine stationäre Rehabilitation indiziert ist oder welche Kriterien für

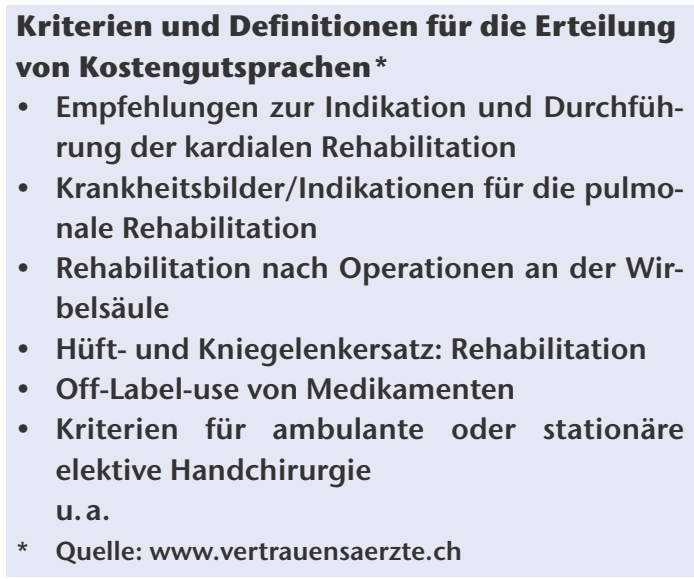

deren ambulante Durchführung sprechen. Auf diese Empfehlungen können sich die Vertrauensärzte bei ihren KoGu-Entscheiden abstützen. Ihre Stellungnahme zuhanden des Versicherers ist jedoch eine Empfehlung, die den Versicherer rechtlich nicht bindet [12].

\section{Stillschweigende Kostengutsprachen - ein Pilotprojekt in Zürich}

Zunehmender bürokratischer Aufwand veranlasste Ärzte des «Zürcher Kreis für fortschrittliches Spitalmanagement», Vorschläge zu dessen Abbau zu formulieren [13] - auch für Kostengutsprachen. An der Herbst-Veranstaltung 2014 der FMH-Fachkommission SwissDRG und FMH-Begleitgruppe ST Reha haben Stefan Wildi [14] und Beat Schärer [15] über erste Projekterfahrungen berichtet. Der hohe Aufwand der Spitäler und Versicherer für Kostengutsprachen wurde deutlich: Rückfragen sowohl spitalintern als auch vom Versicherer, Aufbereitung und Austausch medizinischer Dokumentation sowie das Fehlen konkreter Ansprechpersonen im Spital und beim Versicherer machen die Abläufe komplex. Die Sanitas prüft zudem alle KoGu-Gesuche im Einzelfall, wobei über $90 \%$ genehmigt werden.

Die Spitäler Limmattal, Waid und Triemli haben in Absprache mit dem Vertrauensarzt der Sanitas eine Liste mit Krankheiten erarbeitet, für die in jedem Fall eine stationäre Reha-Behandlung erforderlich ist, und für diese Indikationen eine «stillschweigende Kostengutsprache» vereinbart. Allerdings entsprachen nur wenige der in den drei Spitälern aufgenommenen Fälle genau diesen definierten Voraus- 
setzungen. Eine Vereinfachung im Vergleich zum bekannten KoGu-Verfahren konnte noch nicht festgestellt werden, da u.a. die Rehabilitations-Kliniken eine Bestätigung der Kostenübernahme durch den Versicherer verlangten.

Zwar konnten mit diesem Pilotprojekt noch keine grundlegenden Verbesserungen erzielt werden, aber durch das Wissen über die beidseitigen Arbeitsabläufe und persönliche Ansprechpersonen wurde das Vertrauen zwischen den Beteiligten gestärkt. Die verbesserte Zusammenarbeit spiegelt sich bereits in einer reduzierten Anzahl von Rückfragen wider. Vorgesehen sind nun eine Erweiterung der Indikationsliste für stillschweigende Kostengutsprachen sowie der Einbezug weiterer Versicherer und Reha-Kliniken.

\section{KoGu - denkbare Lösungsansätze aus ärztlicher Sicht}

Problematisch erachten Ärzte die in der KLV enthaltene Regelung, dass es bei jeder stationären Rehabilitation einer vorgängigen KoGu mit ausdrücklicher Bewilligung des Vertrauensarztes bedarf, sowie deren Umsetzung durch die Versicherer. Eine Lösung wäre eine Revision der KLV mit der Änderung der entsprechenden Formulierungen. Dieser Weg ist allerdings langwierig und wurde von den Veranstaltungs-Teilnehmenden nicht favorisiert. Vielmehr sollen Fachgesellschaften und Vertrauensärzte gemeinsam detaillierte und verbindliche Richtlinien erarbeiten, in denen beispielsweise Indikationen und Mindestdauern ärztlicher Leistungen festgelegt sind. Somit könnten KoGu sogar überflüssig werden. Auch aus Sicht der Sanitas werden solche Erweiterungen und Präzisierungen seitens der Fachgesellschaften als wertvoll und für die Vertrauensärzte als sehr hilfreich eingeschätzt.

\begin{tabular}{l} 
Aktuelle Forumthemen \\
$\begin{array}{l}\text { Diskutieren Sie mit! Im Forum präsentieren wir regel- } \\
\text { mässig brisante Themen aus Politik, Ökonomie } \\
\text { und Wissenschaft, die das Schweizer Gesundheitswesen } \\
\text { betreffen. Bringen Sie Ihre Meinung ein oder kom- } \\
\text { mentieren Sie die Äusserungen Ihrer Kolleginnen und } \\
\text { Kollegen. Das Forum finden Sie unter: } \\
\text { www.saez.ch/forum/ }\end{array}$ \\
\hline
\end{tabular}

\section{Referenzen}

1 Damit eine stationäre Behandlung oder Rehabilitation von der OKP übernommen wird, muss der Patient spitalbedürftig sein. Laut Bundesgericht ist Spitalbedürftigkeit gegeben, wenn eine Erkrankung einer akuten Behandlung oder einer stationären Rehabilitation bedarf, aber auch dann, wenn notwendige medizinische Massnahmen nur im Spital zweckmässig oder mit Erfolg durchgeführt werden können und die ambulanten Möglichkeiten erschöpft sind. Für das Vorliegen von Spitalbedürftigkeit können ausser dem Gesundheitszustand auch persönliche Lebensumstände des Patienten ausschlaggebend sein (BGE 126 V 323 E. 2b).

2 Vgl. Art. 3b Krankenpflege-Leistungsverordnung (KLV) und Art. 71a f. Krankenversicherungsverordnung (KVV).

3 Anhang 1 zur KLV, Kapitel 11, Rehabilitation.

4 Art. 8 Abs. 3 Bundesgesetz über die Invalidenversicherung (IVG) i.V.m. Art. 78 Abs. 1 Verordnung über die Invalidenversicherung (IVV).

5 Art. 33 Abs. 1 KVG.

6 Art. 58 Abs. 3 KVG.

7 Entwicklung Rahmenbedingungen Ärzteschaft unter www.fmh.ch $\rightarrow$ Stationäre Tarife $\rightarrow$ Begleitforschung.

8 Ausnahme: Bei der Verlängerung der ärztlichen Psychotherapie nach bereits erfolgten 40 Sitzungen, ist die Kostengutsprache innert 15 Arbeitstagen zu erteilen (Art. 3b Abs. 4 KLV).

9 Art. 29 Abs. 1 Bundesverfassung (BV); BGE 131 V 407 E.1.1.

10 Urteil 1C_372/2014 vom 4.9.2014 E. 6.

11 Urteil 1B_549/2012 vom 12.11.2012 E.2.3.

12 Gebhard Eugster, Rechtsprechung des Bundesgerichts zum KVG, Rz. 4 zu Art. 57, Schulthess Zürich 2010.

13 Manifest «Medizin gegen Spitalbürokratie», Dezember 2011.

14 PD Dr. med. Stefan Wildi, Chefarzt Chirurgische Klinik, Stadtspital Waid, Zürich.

15 Beat Schärer, Leiter Leistungsmanagement und Mitglied der Direktion, Sanitas. 